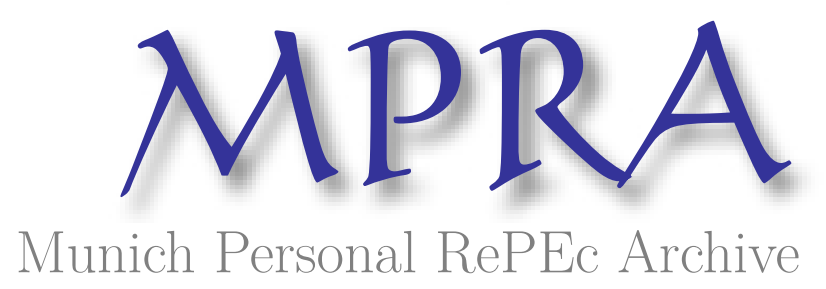

\title{
A multilevel latent Markov model for the evaluation of nursing homes' performance
}

Montanari, Giorgio E. and Doretti, Marco and Bartolucci, Francesco

Department of Political Science, University of Perugia, Department of Political Science, University of Perugia, Department of Economics, University of Perugia

8 August 2017

Online at https://mpra.ub.uni-muenchen.de/80691/

MPRA Paper No. 80691, posted 11 Aug 2017 17:00 UTC 


\title{
A multilevel latent Markov model for the evaluation of nursing homes' performance
}

\author{
Giorgio E. Montanari*1, Marco Doretti ${ }^{1}$, and Francesco Bartolucci ${ }^{2}$ \\ ${ }^{1}$ Department of Political Science, University of Perugia \\ ${ }^{2}$ Department of Economics, University of Perugia
}

\begin{abstract}
August 8, 2017
Abstract

The periodic evaluation of health care services is a primary concern for many institutions. In this work, we focus on nursing home services with the aim to produce a ranking of a set of nursing homes based on their capability to improve - or at least to keep unchanged - the health status of the patients they host. As the overall health status is not directly observable, latent variable models represent a suitable approach. Moreover, given the longitudinal and multilevel structure of the available data, we rely on a multilevel latent Markov model where patients and nursing homes are the first and the second level units, respectively. The model includes individual covariates to account for the patient case-mix and the impact of nursing home membership is modeled through a pair of correlated random effects affecting the initial distribution and the transition probabilities between different levels of health status. Through the prediction of these random effects we obtain a ranking of the nursing homes. Furthermore, the proposed model is designed to address non-ignorable dropout, which typically occurs in these contexts because some elderly patients die before completing the survey. We apply our model to the Long Term Care Facilities dataset, a longitudinal dataset gathered from Regione Umbria (Italy). Our results are robust to the sensitivity parameter involved (the number of latent states) and show that differences in nursing homes' performances are statistically significant.
\end{abstract}

Keywords: clustered data, health status evaluation, non-ingorable dropout, random effects

*The authors gratefully acknowledge Regione Umbria for financial support 


\section{Introduction}

Due to population aging, the demand of health care services from elderly people is constantly increasing in many occidental countries (White, 2007; Gray, 2009). As a consequence, the proper evaluation of the offered services has become a key matter of governments, at national and regional level. Among these services, nursing home care is one of the most relevant (Makai et al., 2014).

In this work, we develop a statistical model to compare the performance of different nursing homes operating in the same context. As an illustration we apply such a model to the health care system of Umbria, a region of central Italy, where a specific public protocol named Long Term Care Facilities (LTCF) has been implemented for many years. As part of the LTCF program, a questionnaire is periodically administered to elderly patients hosted in regional nursing homes (NHs) in order to monitor their overall health status. Thus, a longitudinal dataset is available for many purposes. Specifically, our aim is to use such a database for developing methods of evaluation of the ability of a nursing home to preserve its patients in good health conditions.

For longitudinal multivariate data, latent Markov (LM) models represent an interesting approach when the response variables are categorical and are assumed to measure some underlying characteristic (Bartolucci et al., 2013). This class of models was first introduced by Wiggins (1973) and has become quite popular for this kind of data. In its classical formulation, an LM model assumes that the response variables (typically resulting from the administration of a questionnaire) are affected by an unobserved process evolving over time according to a first-order discrete-time Markov chain with a finite number of states. This unobserved process represents the latent trait of interest, which in our application is the underlying health status of elderly patients. In this sense, an LM model can be 
viewed as the counterpart of the latent class model (Goodman, 1974) for longitudinal data in which each latent class (or latent state) corresponds to a different level of the health status. Many extensions of the basic LM model have been proposed in the literature to account for additional information, represented by individual covariates, or specific data structures; see for example Vermunt et al. (1999). In this paper, we develop a multilevel latent Markov (MLM) model with covariates to deal with the hierarchical structure of the LTCF data (i.e., patients hosted in different nursing homes). The nursing home effect on the health status of their patients is represented by a pair of random effects assumed to affect the distribution of the initial latent state and the transition probabilities between latent states across time. As a matter of fact, our final goal is to rank the nursing homes based on their performance in improving or maintaining their patients' conditions as good as possible.

Though the multilevel extension of the latent class model has been widely discussed and applied in the literature; see, among the others, Vermunt (2003), Henry and Muthén (2010), Montanari et al. (2010), and Gnaldi et al. (2016), its longitudinal counterpart, namely the MLM model, is not as much widespread. To the best of our knowledge, just a few authors have adopted it as a modeling strategy in their applications. For instance, Bartolucci et al. (2011) focus on educational data, while Koukounari et al. (2013) consider the MLM model for the analysis of longitudinal datasets in the medical context. In these papers, the multilevel structure is accounted for by means of cluster-specific time-fixed random effects. Bartolucci and Lupparelli (2016) extend this approach using cluster random effects with a time-varying structure, specifically, a Markov chain. In any case, a discrete distribution over a finite number of support points is specified for the random effects. On the contrary, in this work we adopt a continuous distribution. This strategy permits to obtain one of the outputs of main interest, namely the ranking of second-level units 
(i.e., nursing homes) in a more explicit way with respect to models that, being based on discrete random effects, provide a clustering of such units. There are also advantages in terms of stability of the parameter estimates, being the proposed model more parsimonious than the counterpart based on discrete random effects. Continuous random effects have been already introduced in this field within the so-called class of mixed hidden Markov models (Altman, 2007; Maruotti, 2011; Maruotti and Rocci, 2012). However, often these random effects are intended as means to capture unobserved heterogeneity between units rather than accounting for multilevel data structures.

Bartolucci et al. (2009) propose a similar framework with the aim of evaluating nursing home performances. They also use an LM model with covariates to assess the effect of nursing homes on the probability of transition between latent states through fixed effects introduced by a suitable set of dummy explanatory variables. However, the estimation of these NH effects is rather unreliable - when not unfeasible - if as in our case some NHs contain just a few units. Furthermore, the model proposed here also accounts for dropout due to the death of patients, which is a common problems arising in this type of applications. Ignoring the missing data mechanism may lead to biased estimates. Overall, up to our knowledge, the application of an MLM model to the present context (multivariate longitudinal data with missing values) is innovative.

The outline of the paper is as follows. In Section 2 we present in detail the LTCF data considered in our analysis. The proposed MLM model is illustrated in Section 3, while model results are reported in Section 4. Main conclusions are given in Section 5 together with suggestions for future research in this field. 


\section{The LTCF dataset}

The data motivating the proposed approach come from the Suite interRAI questionnaire, an internationally validated and widely adopted tool (Hirdes et al., 2008; Kim et al., 2015). Our sample refers to the years 2012 and 2013 and contains 1,292 individuals grouped in 47 different NHs. The questionnaire is planned to be administered approximately every six months so that, ideally, four measurement occasions - one for each semester of the two years - should be present for each patient. However, only 3,924 instead of $5,168(4 \times 1,292)$ observations are available. This is due to either intermittent missingness, when a patient does not respond at a given measurement occasion but responds at a following occasion, or dropout, due to patients leaving the study before scheduled because of death or other causes. Intermittent missingness involves a modest, though not negligible, proportion of observations (204, approximately 5\%). Dropout has a more severe impact as it concerns 439 individuals (34\% of the sample). About the reasons for dropout, death occurs in 377 cases $(86 \%)$; other reasons are discharge or transfer to other structures such as hospitals. A description of how such missingness mechanisms are taken into account is provided in Section 3.2.

We have to note that, although observations of each patient are expected to be collected every six months, the time intervals between observations show a variability related to the evolution of patients' health conditions. Observations are sometimes anticipated in the presence of some change in these conditions, or delayed in the opposite case.

The entire Suite interRAI questionnaire is divided in several sections referring to different spheres of the health status, which in general is a multidimensional phenomenon. However, in this paper we focus on a single section of the questionnaire. This section is an important one as it deals with the Activities of Daily Living (ADL), that is, it measures 
the difficulty patients experience in taking common actions. Specifically, the ADL section includes ten items, which are described in Table 1. Focussing only on the ADL implies that the latent trait we consider represents just patients' physical condition in a strict sense. This approach might be perceived as limiting, because other important aspects of the health status (cognitive conditions, humoral status, etc.) are not included in the analysis. However, it permits to represent the latent trait as a unidimensional variable having a meaningful interpretation.

The response variables are measured on an ordinal scale with 6 categories (1-6), from no difficulty at all to complete dependence on other people. In the upper part of Table 1, for each ADL item we report the frequency distribution of the response categories referred to all the 3,924 observations. In the bottom-left-hand side we report the item average response (i.e., the mean of the category labels weighted with their frequencies) for each time occasion $t=1, \ldots, 4$. Note that, although one expects an increase in the ADL difficulties over time, this is not the case because of dropout. In the bottom-right-hand side of Table 1, we also report for each time the average patients' age $\left(X_{1}^{(t)}\right)$, the proportion of females $\left(X_{2}^{(t)}\right)$, the average time interval in days between the current and the previous observation $\left(X_{3}^{(t)}\right)$, the

number of patients with $t$ observations $\left(m_{1}^{(t)}\right)$, and the number of patients surveyed at the t-th occasion $\left(m_{2}^{(t)}\right)$.

\section{The multilevel latent Markov model}

In this section, we present the MLM model. Here, we introduce the main notation, while the model specification, including how the missing data mechanism is formulated, is illustrated in Sections 3.1 and 3.2. Finally, Section 3.3 describes the maximum likelihood estimation of the proposed model. 


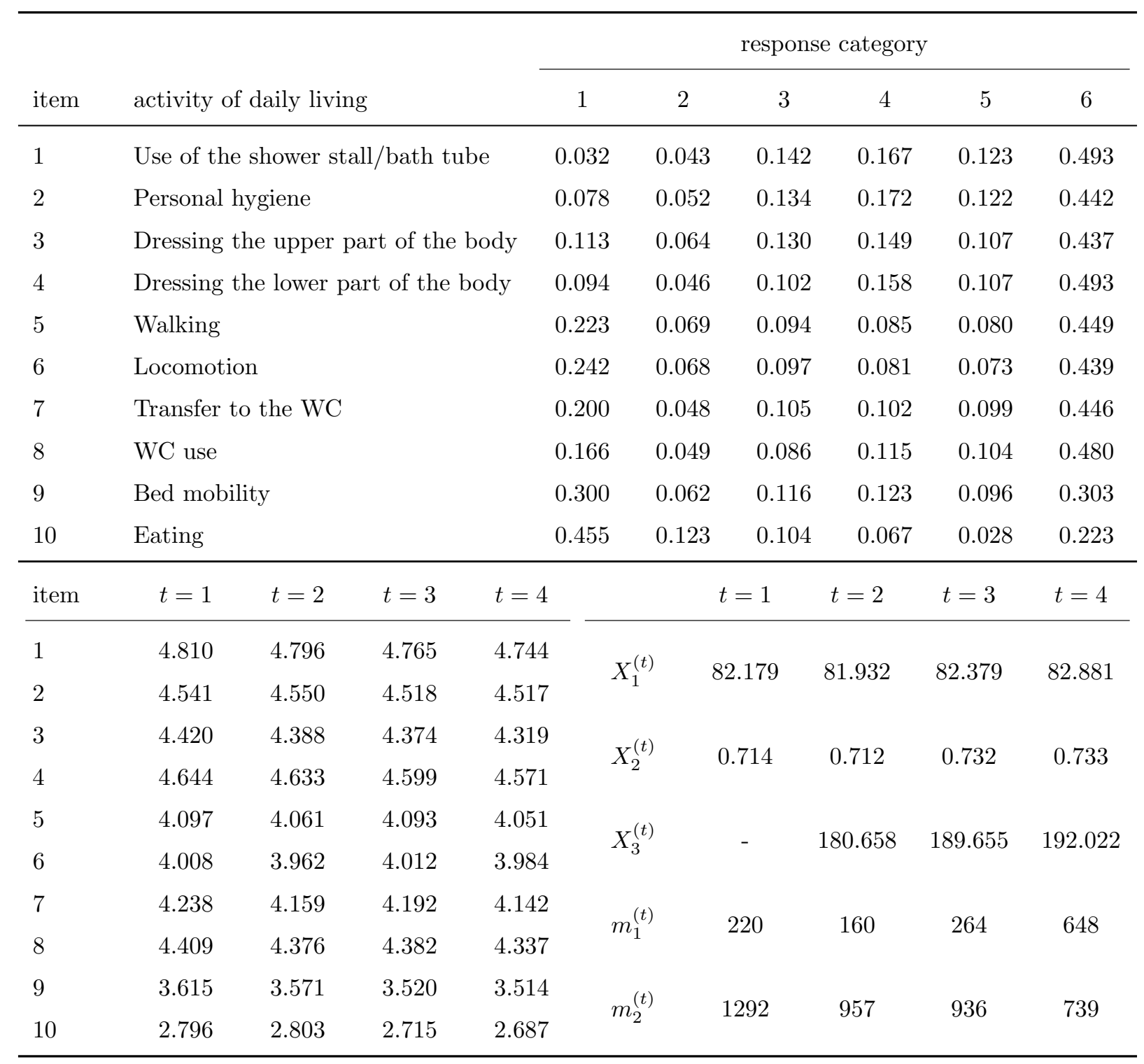

Table 1: Description and frequency distributions (3,924 observations) of items in the ADL section. Average values of response items and covariates at different time occasions. $X_{1}^{(t)}$ : age (years), $X_{2}^{(t)}$ : gender (1=female), $X_{3}^{(t)}$ : distance from previous occasion (days). $m_{1}^{(t)}$ : number of patients with $t$ measurement occasions, $m_{2}^{(t)}$ : number of patients at the $t$-th occasion 
As typical in a multilevel analysis, we have $n$ sample units divided into $H$ different clusters. Therefore, every unit is characterized by a double index $h i$, with $h=1, \ldots, H$ and $i=1, \ldots, n_{h}$, where $n_{h}$ is the dimension of the $h$-th cluster so that $n=\sum_{h=1}^{H} n_{h}$. Furthermore, we denote by $\boldsymbol{Y}_{h i}^{(t)}$ the response vector of unit $i$ in cluster $h$ at occasion $t$. This vector consists of $J$ univariate categorical responses, that is $\boldsymbol{Y}_{h i}^{(t)}=\left(Y_{h i 1}^{(t)}, \ldots, Y_{h i J}^{(t)}\right)$. Each univariate response $Y_{h i j}^{(t)}$ might have a generic number of categories $c_{j}, j=1, \ldots, J$. Moreover, every unit $h i$ can have a specific number of measurement occasions $T_{h i} \leq T$, where $T$ denotes the maximum number of occasions observed in the sample for a unit.

The vectors $\boldsymbol{Y}_{h i}^{(t)}$ can be collected across time in the vector $\boldsymbol{Y}_{h i}=\left(\boldsymbol{Y}_{h i}^{(1)}, \ldots, \boldsymbol{Y}_{h i}^{\left(T_{h i}\right)}\right)$. In turn, we let $\boldsymbol{Y}_{h}=\left(\boldsymbol{Y}_{h 1}, \ldots, \boldsymbol{Y}_{h n_{h}}\right)$ be the vector of all observations of cluster $h$. Individual covariates are denoted by $\boldsymbol{X}_{h i}^{(t)}$, and similarly to the response variables we set $\boldsymbol{X}_{h i}=$ $\left(\boldsymbol{X}_{h i}^{(1)}, \ldots, \boldsymbol{X}_{h i}^{\left(T_{h i}\right)}\right)$ and $\boldsymbol{X}_{h}=\left(\boldsymbol{X}_{h 1}, \ldots, \boldsymbol{X}_{h n_{h}}\right)$. Every individual-specific Markovian latent process is denoted by $\boldsymbol{V}_{h i}=\left(V_{h i}^{(1)}, \ldots, V_{h i}^{\left(T_{h i}\right)}\right)$, where, at each time occasion $t, V_{h i}^{(t)}$ is a categorical variable with $k$ levels. Again, individual latent processes within the same cluster can be collected in the vector $\boldsymbol{V}_{h}=\left(\boldsymbol{V}_{h 1}, \ldots, \boldsymbol{V}_{h n_{h}}\right)$. Finally, we denote the vector of cluster-specific random effects, which are time invariant, with $\boldsymbol{U}_{h}=\left(W_{h}, Z_{h}\right)$.

\subsection{Model formulation}

In the MLM model, the latent processes of two different clusters - say $\boldsymbol{V}_{h}$ and $\boldsymbol{V}_{h^{\prime}}$ - are considered independent. As a consequence, the vectors $\boldsymbol{Y}_{1}, \ldots, \boldsymbol{Y}_{H}$ collecting the responses at cluster level are marginally independent. However, for units in the same cluster $h$, the independence is conditional on the cluster random effect $\boldsymbol{U}_{h}$. Moreover, the Markovian structure governing the latent individual processes $\boldsymbol{V}_{h i}$ is assumed to hold conditionally on the cluster random effect $\boldsymbol{U}_{h}$ and on individual covariates $\boldsymbol{X}_{h i}$. This means that the conditional initial and first order transition probabilities fully determine such a latent 
process. Specifically, we define the conditional initial probabilities as

$$
\pi_{h i}^{(1)}(v)=P\left(V_{h i}^{(1)}=v \mid \boldsymbol{X}_{h i}^{(1)}=\boldsymbol{x}_{h i}^{(1)}, W_{h}=w_{h}\right), \quad v=1, \ldots, k,
$$

and the conditional transition probabilities as

$$
\pi_{h i}^{(t)}(v \mid \bar{v})=P\left(V_{h i}^{(t)}=v \mid V_{h i}^{(t-1)}=\bar{v}, \boldsymbol{X}_{h i}^{(t)}=\boldsymbol{x}_{h i}^{(t)}, Z_{h}=z_{h}\right), \quad v, \bar{v}=1, \ldots, k, t=2, \ldots, T_{h i},
$$

where $W_{h}$ and $Z_{h}$ are the two components of $\boldsymbol{U}_{h}$ already defined above. Notice that we are implicitly assuming that $Z_{h}$ is independent of $V_{h i}^{(1)}$ given $\left(\boldsymbol{X}_{h i}^{(1)}, W_{h}\right)$ and that $W_{h}$ is independent of $V_{h i}^{(t)}$ given $\left(V_{h i}^{(t-1)}, \boldsymbol{X}_{h i}^{(t)}, Z_{h}\right)$ for $t=2, \ldots, T_{h i}$. Also, $\boldsymbol{U}_{h}$ is assumed to be marginally independent of $\boldsymbol{X}_{h}$. Such conditional initial and transition probabilities are collected into individual-specific vectors

$$
\boldsymbol{\pi}_{h i}^{(1)}=\left(\pi_{h i}^{(1)}(1), \ldots, \pi_{h i}^{(1)}(k)\right)
$$

and matrices

$$
\Pi_{h i}^{(t)}=\left(\begin{array}{ccc}
\pi_{h i}^{(t)}(1 \mid 1) & \ldots & \pi_{h i}^{(t)}(k \mid 1) \\
\vdots & \ddots & \vdots \\
\pi_{h i}^{(t)}(1 \mid k) & \ldots & \pi_{h i}^{(t)}(k \mid k)
\end{array}\right), \quad t=2, \ldots, T_{h i} .
$$

Their dependence on cluster membership and individual covariates is modeled by the regression equations

$$
\log \frac{\pi_{h i}^{(1)}(v+1)+\cdots+\pi_{h i}^{(1)}(k)}{\pi_{h i}^{(1)}(1)+\cdots+\pi_{h i}^{(1)}(v)}=\xi_{v}+\boldsymbol{x}_{h i}^{(1)} \boldsymbol{\beta}+w_{h} \sigma_{w}, \quad v=1, \ldots, k-1
$$

and $\log \frac{\pi_{h i}^{(t)}(v+1 \mid \bar{v})+\cdots+\pi_{h i}^{(t)}(k \mid \bar{v})}{\pi_{h i}^{(t)}(1 \mid \bar{v})+\cdots+\pi_{h i}^{(t)}(v \mid \bar{v})}=\psi_{\bar{v}}+\omega_{v}+\boldsymbol{x}_{h i}^{(t)} \gamma+z_{h} \sigma_{z}, \quad v=1, \ldots, k-1, \bar{v}=1, \ldots, k$, 
for $t=2, \ldots, T_{h i}$.

In Equations (1) and (2), a global logit parametrization is assumed; see Bartolucci et al. (2009) for more details about this parametrization applied in a similar context. Under this parametrization, the covariate effects, represented by the column vectors $\boldsymbol{\beta}$ and $\boldsymbol{\gamma}$, are constant across the logit equations, while the sequences of thresholds $\xi_{1} \geq \cdots \geq \xi_{k-1}$ and $\omega_{1} \geq \cdots \geq \omega_{k-1}$ must be non-increasing to ensure that the cumulative sums of probabilities along the ordered categories of the latent variables are non-decreasing. On the contrary, no order restrictions are posed on the sequence $\psi_{1}, \ldots, \psi_{k}$. However, $\psi_{1}=0$ is set for identification purposes.

A standard bivariate normal distribution with correlation coefficient $\rho$ is assumed for the cluster effect random vector $\boldsymbol{U}_{h}$. The overall variability of the clustering process is governed by the parameters $\sigma_{w}$ and $\sigma_{z}$ in (1) and (2). These parameters are obviously constrained to be non-negative in the estimation phase (see Section 3.3). The higher (and the more statistically significant) their deviations from zero, the higher the relevance of clustering in the data (and therefore the necessity to account for it).

Individual covariates and cluster effects are assumed not to enter in the measurement model, that is, in the model for the outcomes given the latent process. In the present context, this assumption allows to interpret the latent states $v=1, \ldots, k$ as different levels of seriousness of patients' conditions. As a matter of fact, each outcome $Y_{h i j}^{(t)}$ is assumed to be independent of any other variable in the model conditionally on $V_{h i}^{(t)}$. Under this setting, the relevant parameters are the conditional response probabilities

$$
\phi_{j y_{j} v}=P\left(Y_{h i j}^{(t)}=y_{j} \mid V_{h i}^{(t)}=v\right),
$$

with $j=1, \ldots, J, y_{j}=1, \ldots, c_{j}$, and $v=1, \ldots, k$. Notice that these probabilities are not indexed by $t$ and therefore are assumed constant with respect to time. For each outcome, 
the conditional response probabilities can be stored in the matrix

$$
\boldsymbol{\Phi}_{j}=\left(\begin{array}{ccc}
\phi_{j 11} & \ldots & \phi_{j c_{j} 1} \\
\vdots & \ddots & \vdots \\
\phi_{j 1 k} & \ldots & \phi_{j c_{j} k}
\end{array}\right), \quad j=1, \ldots, J .
$$

However, the number of unconstrained conditional response probabilities is rather high even in relatively small settings.

In order to make the model more parsimonious, several different parametrizations can be imposed. Also in this case, we adopt a global logit parametrization of type

$$
\log \frac{\phi_{j, m+1, v}+\cdots+\phi_{j c_{j} v}}{\phi_{j 1 v}+\cdots+\phi_{j m v}}=\tau_{j m}+\delta_{v}
$$

for $j=1, \ldots, J, m=1, \ldots, c_{j}-1$, and $v=1, \ldots, k$. As in (1) and (2), in Equation (3) the sequences of thresholds $\tau_{j 1} \geq \cdots \geq \tau_{j, c_{j}-1}$ must be non-increasing for $j=1, \ldots, J$. Moreover, $\delta_{1}$ is set to 0 to ensure model identifiability and $\delta_{1} \leq \cdots \leq \delta_{k}$ is imposed in order to obtain a positive association between the responses and the latent variable. In this way, we can tackle the label switching problem, that is typical of discrete latent variable models (see, e.g., Stephens, 2000). Notice that this parametrization provides a

clear interpretation of the latent states and is appropriate as the latent variables $V_{h i}^{(t)}$ have an ordinal nature.

\subsection{Missing data modelling}

As already shown in Section 2, in the LTCF dataset we consider there is a relatively large proportion of missing data. Therefore, a careful evaluation of the missingness mechanism is needed. On one hand, intermittent missingness and dropout due to reasons other than death do not have a well-established relationship with patients' physical health status. On 
the other hand, dropout due to death is very often associated to a worsening in patients' health conditions. Therefore, the former can be treated as ignorable (Little and Rubin, 2002), while the latter clearly cannot, and an explicit model needs to be set for it.

Dropout due to death is modeled by expanding the available set of observations. Specifically, for each response $j$, we add an extra response category $c_{j}+1$ in a way such that data trajectories for patients dead after the $t$-th occasion $(t<T)$ are completed by setting $Y_{h i j}^{(u)}=c_{j}+1$ for $u=t+1, \ldots, T$. A similar approach was undertaken also by Montanari and Pandolfi (2016). In our application, the total number of observations raises from 3,924 to 4,746 after this expansion. We also define an extra latent state $k+1$ corresponding to death, which may be seen as an extreme health condition. Some of the extra probabilities generated by this adjustment are suitably constrained. Specifically, we have:

- $\pi_{h i}^{(1)}(k+1)=0$ for all $h$ and $i$ : no one can be in the extra death state at the first occasion;

- $\pi_{h i}^{(t)}(v \mid k+1)=0$ and $\pi_{h i}^{(t)}(k+1 \mid k+1)=1$ for all $h$ and $i$, and $t=2, \ldots, T$ : no one can revert to other latent states after being in the extra death state (also called absorbing state);

- $\phi_{j, c_{j}+1, v}=0, v=1, \ldots, J$, and $\phi_{j, c_{j}+1, k+1}=1$ for all $j$ : only the extra response category can be observed if one is in the extra latent state.

Thus, initial probability vectors and transition matrices take the form $\boldsymbol{\pi}_{h i}^{(1)}=\left(\pi_{h i}^{(1)}(1), \ldots, \pi_{h i}^{(1)}(k), 0\right), \quad \Pi_{h i}^{(t)}=\left(\begin{array}{cccc}\pi_{h i}^{(t)}(1 \mid 1) & \ldots & \pi_{h i}^{(t)}(k \mid 1) & \pi_{h i}^{(t)}(k+1 \mid 1) \\ \vdots & \ddots & \vdots & \vdots \\ \pi_{h i}^{(t)}(1 \mid k) & \ldots & \pi_{h i}^{(t)}(k \mid k) & \pi_{h i}^{(t)}(k+1 \mid k) \\ 0 & 0 & 0 & 1\end{array}\right)$, 
while each conditional response matrix becomes

$$
\boldsymbol{\Phi}_{j}=\left(\begin{array}{cccc}
\phi_{j 11} & \ldots & \phi_{j c_{j} 1} & 0 \\
\vdots & \ddots & \vdots & 0 \\
\phi_{j 1 k} & \ldots & \phi_{j c_{j} k} & 0 \\
0 & 0 & 0 & 1
\end{array}\right) .
$$

Clearly, $\pi_{h i}^{(t)}(k+1 \mid 1), \ldots, \pi_{h i}^{(t)}(k+1 \mid k)$ are the only unconstrained additional probabilities our dropout correction entails to estimate. These are the probabilities of transition to the death state, given the patient is in a generic latent state $v$ at the previous occasion, for $v=$ $1, \ldots, k$. According to the adopted parametrization, this corresponds to the estimation of the single additional threshold $\omega_{k}$, with $\omega_{k} \leq \omega_{k-1}$ : the probabilities which are constrained to zero or one are obtained by introducing new thresholds taking values equal to $-\infty$ or $+\infty$, respectively. Finally, the model extension we propose to deal with missing data involves only one free parameters to estimate: the additional threshold $\omega_{k}$.

\subsection{Maximum likelihood estimation}

Denoting by $p_{1}=|\boldsymbol{\beta}|$ and $p_{2}=|\boldsymbol{\gamma}|$ the number of individual covariates affecting the initial and transition probabilities, the total number of free model parameters is

$$
p=4 k+p_{1}+p_{2}+\sum_{j=1}^{J}\left(c_{j}-1\right) .
$$

Specifically, we have:

- $k-1+p_{1}$ parameters for the initial probabilities;

- $2 k-1+p_{2}$ parameters for the transition probabilities; 
- $\sum_{j=1}^{J}\left(c_{j}-1\right)+k-1$ parameters for the conditional response probabilities;

- 3 parameters for the cluster random effect.

Putting all these parameters in a single vector $\boldsymbol{\theta}$, the model log-likelihood is expressed by

$$
\ell(\boldsymbol{\theta})=\sum_{h=1}^{H} \log P\left(\boldsymbol{Y}_{h}=\boldsymbol{y}_{h} \mid \boldsymbol{X}_{h}=\boldsymbol{x}_{h}\right) .
$$

Computing the above log-likelihood obviously requires the computation of the clusterspecific probabilities $P\left(\boldsymbol{Y}_{h}=\boldsymbol{y}_{h} \mid \boldsymbol{X}_{h}=\boldsymbol{x}_{h}\right)$. The model assumptions imply that

$$
P\left(\boldsymbol{Y}_{h}=\boldsymbol{y}_{h} \mid \boldsymbol{X}_{h}=\boldsymbol{x}_{h}\right)=\int_{\mathbb{R}^{2}}\left[\prod_{i=1}^{n_{h}} P\left(\boldsymbol{Y}_{h i}=\boldsymbol{y}_{h i} \mid \boldsymbol{X}_{h i}=\boldsymbol{x}_{h i}, \boldsymbol{U}_{h}=\boldsymbol{u}_{h}\right)\right] \phi\left(\boldsymbol{u}_{h} ; \mathbf{0}, \boldsymbol{\Sigma}\right) d \boldsymbol{u}_{h},
$$

where $\phi\left(\boldsymbol{u}_{h} ; \mathbf{0}, \boldsymbol{\Sigma}\right)$ denotes the density function at $\boldsymbol{u}_{h}$ of a bivariate normal distribution with null mean and variance-covariance matrix

$$
\Sigma=\left(\begin{array}{ll}
1 & \rho \\
\rho & 1
\end{array}\right)
$$

with $\rho$ being the correlation between the two components $W_{h}$ and $Z_{h}$ of $\boldsymbol{U}_{h}$. For every $h i$, $P\left(\boldsymbol{Y}_{h i}=\boldsymbol{y}_{h i} \mid \boldsymbol{X}_{h i}=\boldsymbol{x}_{h i}, \boldsymbol{U}_{h}=\boldsymbol{u}_{h}\right)$ may be computed by the Baum-Welch forward recursion (Baum et al., 1970; Welch, 2003). To compute the integral in (5), we rely on a bivariate quadrature approximation (Press et al., 1989) based on the following steps. First, by a Gauss-Hermite method we define $Q$ quadrature points $\boldsymbol{\nu}_{q}^{*}$ and the corresponding weights $\lambda_{q}^{*}(q=1, \ldots, Q)$ for the standard bivariate normal distribution (with $\left.\rho=0\right)$. Then, to account for the correlation $\rho$ we let

$$
\boldsymbol{\nu}_{q}=\boldsymbol{L} \boldsymbol{\nu}_{q}^{*}, \quad \lambda_{q}=\lambda_{q}^{*}, \quad q=1, \ldots, Q,
$$

where $\boldsymbol{L}$ is the Choleski decomposition of $\boldsymbol{\Sigma}$. Finally, we use the approximation

$$
P\left(\boldsymbol{Y}_{h}=\boldsymbol{y}_{h} \mid \boldsymbol{X}_{h}=\boldsymbol{x}_{h}\right) \approx \sum_{q=1}^{Q}\left[\prod_{i=1}^{n_{h}} P\left(\boldsymbol{Y}_{h i}=\boldsymbol{y}_{h i} \mid \boldsymbol{X}_{h i}=\boldsymbol{x}_{h i}, \boldsymbol{U}_{h}=\boldsymbol{\nu}_{q}\right)\right] \lambda_{q} .
$$


In our application, we consider 11 quadrature points for each dimension, so that a grid of $Q=11^{2}=121$ points is obtained overall. This approximation is reasonable and higher values would result in prohibitive computational times.

Parameter estimates $\hat{\boldsymbol{\theta}}$ are obtained from direct maximization of the log-likelihood. For this aim we rely on a BFGS algorithm (Fletcher, 1987), which is available in the statistical software R. In order to run this algorithm, the approximate log-likelihood gradient must be calculated; see Supplementary material Section 1 for details on how to compute this gradient. The BFGS algorithm also returns the Hessian matrix containing the numerical second derivatives of $\ell(\boldsymbol{\theta})$ at the maximum likelihood estimate $\hat{\boldsymbol{\theta}}$. Therefore, the estimated variance-covariance matrix is readily available to compute standard errors for the parameter estimates.

In the end it is worth clarifying some aspects about the implementation of the maximum likelihood estimation. First of all, the whole maximization algorithm is run using suitable transformations of the random effect parameters $\sigma_{w}, \sigma_{z}$, and $\rho$. Specifically, logarithmic transformations for $\sigma_{w}$ and $\sigma_{z}$ are introduced to ensure these parameters are positive (see Section 3.1). Moreover, a Fisher transformation $F(\rho)=1 / 2 \log [(1+\rho) /(1-\rho)]$ is used for $\rho$ so that its final estimate is ensured to lie between -1 and 1 (Fisher, 1915). The variancecovariance matrix of these parameters on the original scale, namely prior to transformations, can be easily approximated by using the Delta method (Oehlert, 1992). Moreover, as any iterative algorithm, the BFGS needs to start from an initial guess for the parameter vector $\boldsymbol{\theta}$. In this regard we recall that for complex latent variable models, as that object of the present paper, multimodality of the likelihood function may arise and this leads to a lack of uniqueness of the maximum likelihood solution. A typical solution consists in trying different initializations for the estimation algorithm. In our application, we mainly follow a strategy based on using sensible starting values based on a preliminary estimation of an 
LM model with cluster-level fixed effects (Bartolucci et al., 2009), which is performed by an Expectation Maximization algorithm (Dempster et al., 1977), and letting $\sigma_{w}=\sigma_{z}=1$ and $\rho=0$ as starting values. In fact, given the complexity of the model, trying many different starting values may be prohibitive in terms of computing time for large values of $k$. In any case, we tried different starting values which were randomly generated and we found that the initial solution based on the preliminary fitting of the fixed-effects LM model is typically better than other solutions.

\section{Results}

In this section, we summarize the results from the fitting of the MLM model on the LTCF data. We recall that in this dataset $n=1,292$ patients are divided in $H=47$ NHs. Moreover, there are $J=10$ categorical outcomes with the same number of categories, that is, $c_{j}=c=6$ for $j=1, \ldots, J$. Finally, because of intermittent missing data and dropout we have a varying number of measurement occasions $T_{h i} \leq T=4$ for each patient. Age, age squared, gender, and time interval between occasions are considered as individual covariates (see Section 2). Clearly, the last covariate covariate is included only in the model for transition probabilities (see Equation (2)) and not in the model for initial probabilities. In this way we account for the variability of the time length between consecutive time occasions for the same individual. Typically, one is interested in six-month ahead transition probabilities, as six months is the interval between measurements originally designed in the LTCF study, though deviations from this target are observed.

In the reminder of this section, we first provide an outline of the procedure we adopted for model selection (Section 4.1). Then, results for the selected models are reported (Section 4.2). 


\subsection{Model selection}

As typical in the latent variable framework, model selection is essentially concerned with the choice of the number of latent states $k$. This decision requires a preliminary exploration of a range of different values of $k$. In this application, we fit models with $k$ from 2 to 7 . We avoid higher values of $k$ as it is known that models with many latent states often result in some of the generated subgroups having a reduced dimension and collecting sample units with a behavior very far different from the majority of the sample. Typically, these spurious subgroups do not represent real segments of the population under investigation, and their existence is due to overfitting only. Moreover, for $k>7$ the threshold constraints of Section 3.1 are unlikely to be met without additional model structure.

One way to account for model complexity is to rely on classical information criteria based on the log-likelihood penalization. Specifically, here for every model we compute the Bayesian Information Criterion (BIC, Schwarz, 1978), defined as

$$
B I C=-2 \ell(\hat{\boldsymbol{\theta}})+p \log n,
$$

where $n$ is the number of sample units and $p$ is the overall number of free parameters. In principle, models with a lower $B I C$ should be preferred. However, this index is often prone to favor models with too many latent states; see Bacci et al. (2014) for a detailed comparison of different selection criteria in a similar context. Therefore, it is a good practice to also consider alternative criteria. For instance, one could assess how sharp the posterior (i.e., given the data) classification of units into the latent states is. Formally, letting

$$
\tilde{v}_{h i}^{(t)}=\max _{v=1, \ldots, k} P\left(V_{h i}^{(t)}=v \mid \boldsymbol{X}_{h i}=\boldsymbol{x}_{h i}, \boldsymbol{U}_{h}=\boldsymbol{u}_{h}, \boldsymbol{Y}_{h i}=\boldsymbol{y}_{h i}\right),
$$




\begin{tabular}{ccccccc}
\hline$k$ & 2 & 3 & 4 & 5 & 6 & 7 \\
\hline$B I C$ & $92,486.98$ & $81,888.21$ & $77,430.13$ & $76,010.54$ & $75,393.62$ & $74,924.52$ \\
$G$ & 0.9896 & 0.9796 & 0.9729 & 0.9578 & 0.9476 & 0.9524 \\
\hline
\end{tabular}

Table 2: Values of $B I C$ and $G$ for models with $k \in 2, \ldots, 7$

for the MLM a measure of the sharpness of a $k$-state model's classification is given by

$$
G=\frac{\sum_{h=1}^{H} \sum_{i=1}^{n_{h}} \sum_{t=1}^{T_{h i}}\left(\tilde{v}_{h i}^{(t)}-\frac{1}{k}\right)}{N\left(1-\frac{1}{k}\right)}
$$

with $N$ being the total number of observations. The $G$ index varies between 0 and 1 , with 0 corresponding to random classification (i.e., units classified with constant probability $1 / k$ for every state) and 1 corresponding to the sharpest classification (that is, one latent state has probability 1). Notice that to compute $G$ we here focus only on the original 3,924 observations instead of the 4,746 records obtained after the data expansion due to accounting fro dropout. Indeed, for the additional observations the latent process is in the extra latent state $k+1$ with probability 1 by construction.

Table 2 reports the $B I C$ and the $G$ index for the considered models. As expected, the $B I C$ tends to support models with a higher number of states, while all values of $G$ are close to 1 , denoting a good classification capability for all values of $k$. According to Table 2, one might think the model with $k=7$ represents a good compromise. Nevertheless, results from this model show that the second latent state contains only the $2 \%$ of patients and has the same conditional response probabilities of state 3 . The only relevant difference is in the average transition probability which is 0.863 for state 2 and zero for state 3 . Overall, this is likely to be a case of overfitting as described above. As a consequence, we prefer to consider results from models with five and six latent states. In the following subsection we 
describe the results obtain from the former, while the results under latter, which are not substantially different, are reported in the Supplementary material, Section 2.

\subsection{Estimation results for $k=5$}

The five latent states may be characterized in terms of conditional response probabilities. A summary picture can be provided by the normalized item scores

$$
s_{j v}=\frac{1}{c_{j}-1} \sum_{y_{j}=1}^{c_{j}}\left(y_{j}-1\right) \hat{\phi}_{j y_{j} v} \quad v=1, \ldots, k, \quad j=1, \ldots, J .
$$

These scores vary in the 0-1 interval and measure the difficulty a patient in latent state $v$ experiences in taking the action associated to item $j$. A value near to zero indicates that most of the conditional probability is attributed to the first category, corresponding to no difficulty at all in the activity of daily living the item refers to. On the contrary, a value near to one means that the probability is mostly allocated in the last category, corresponding to totally unable to do the activity.

The normalized item scores for each latent state are plotted in Figure 1; see Table 1 for a summary description of each item. Patients in the first latent state experience some difficulties and require some assistance related to the first four items: use of the shower stall or bath tube, personal hygiene, and dressing the upper, and lower part of the body.

The need for assistance increases for individuals in the second latent state with respect to the activities related to those items and some initial difficulties arise in transferring to and using the WC, walking, and moving around. For patients in latent state 3, the needed assistance become intensive for all activities apart from mobility in bed and eating. Latent state 4 includes those requiring the assistance of two or more persons (apart from eating, for which the assistance is limited). Finally, individuals in latent state 5 are totally unable with respect to activities related to all items. 


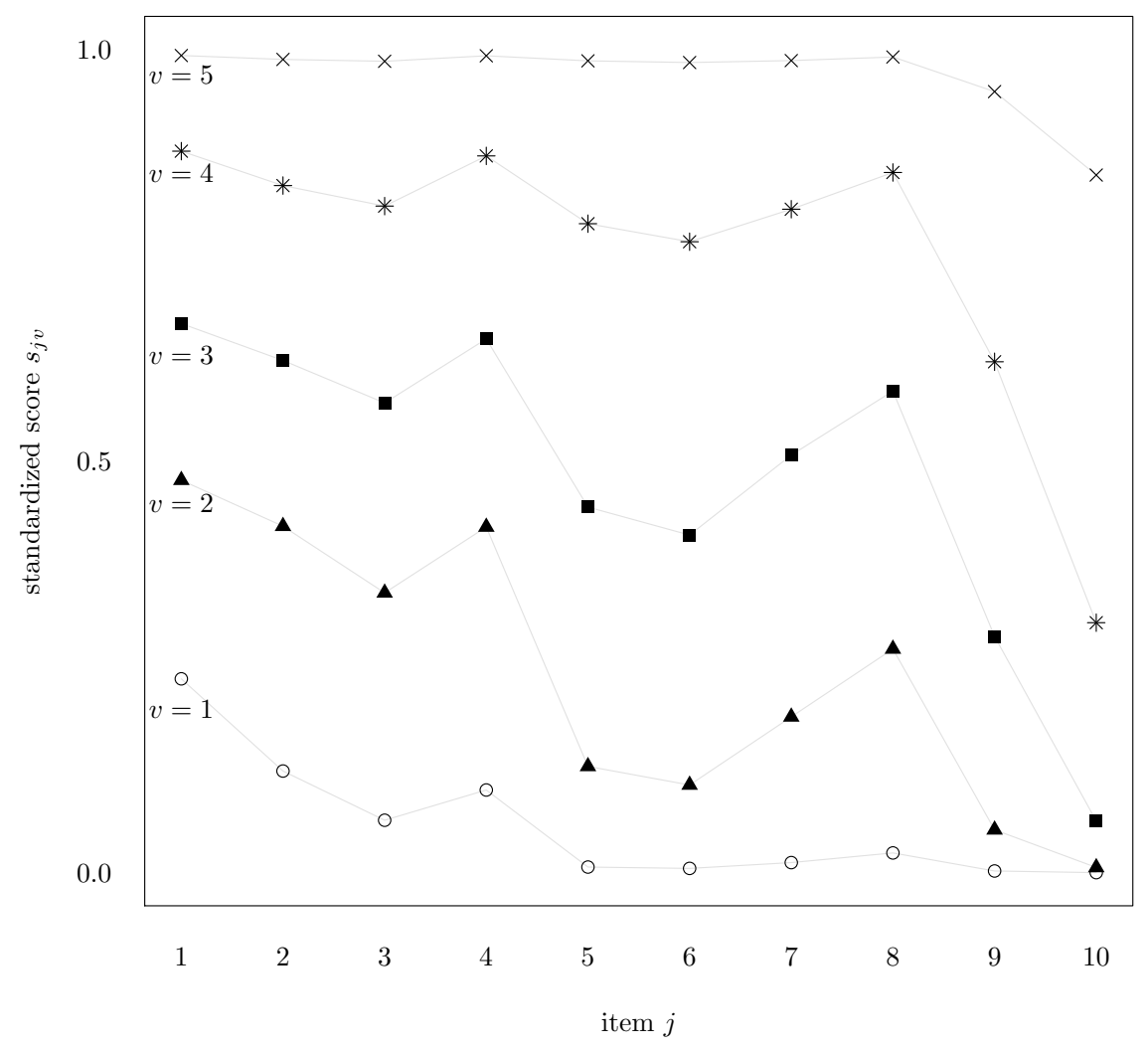

Figure 1: Normalized item scores $s_{j v}(k=5)$ 
Turning the attention to the latent process, Table 3 contains estimates and standard errors for the main parameters of the model with five latent states, once the covariate age squared - that is not significant - has been removed. The coefficients $\hat{\beta}_{1}$ and $\hat{\gamma}_{1}$ represent the effect of patients' age (in years) on their initial and transition probabilities respectively.

Given the assumed parametrization, it is not surprising to observe positive values, meaning that older patients have initial and transition probabilities more concentrated on the higher states, that is on the states associated to worse health conditions. It is also interesting to examine the gender effect, expressed by $\hat{\beta}_{2}$ and $\hat{\gamma}_{2}$. As gender is coded here as a binary variable equal to 1 for females and 0 for males, from Table 3 we evince that females are in a worse physical condition at the first occasion $\left(\hat{\beta}_{2}>0\right)$, whereas males migrate towards critical health states with higher probability at the following occasions $\left(\hat{\gamma}_{2}<0\right)$. Finally, the negative estimate $\hat{\gamma}_{3}$ denotes that patients for which measurement occasions are more distant have a lower tendency to move to worse states. This result may be explained by nursing homes' tendency to anticipate measurements when there is a worsening in the health conditions of their patients (see Section 2). All these parameters are significantly different from zero at 5\% significance level. A significant effect of age on initial probabilities, consistent with the one reported here, is found also by Bartolucci et al. (2009), which focus on the period 2003-2005. However, their findings exclude a significant

\begin{tabular}{ccccccccc}
\hline par. & $\beta_{1}$ & $\beta_{2}$ & $\gamma_{1}$ & $\gamma_{2}$ & $\gamma_{3}$ & $\sigma_{w}$ & $\sigma_{z}$ & $\rho$ \\
\hline est. & 0.032 & 0.503 & 0.019 & -0.267 & -0.004 & 0.461 & 0.384 & -0.118 \\
s.e. & 0.006 & 0.121 & 0.004 & 0.096 & 0.001 & 0.098 & 0.056 & 0.221 \\
\hline
\end{tabular}

Table 3: Main parameter estimates and standard errors for the model with $k=5$ 
effect of gender on initial and transition probabilities as well as an effect of age on transition probabilities.

The two random effect coefficients are $\hat{\sigma}_{w}=0.461$ and $\hat{\sigma}_{z}=0.384$, whereas the estimated correlation is $\hat{\rho}=-0.118$. Standard errors for these estimates are reported in Table 3, but statistical $t$-tests are performed on the parameter transformation scales mentioned in Section 3.3 to deal with parameters varying on the real line. Specifically, we have $\log \left(\hat{\sigma}_{w}\right)=-0.774$ (s.e. 0.212 ), $\log \left(\hat{\sigma}_{z}\right)=-0.957$ (s.e. 0.146 ), and $F(\hat{\rho})=-0.119$ (s.e. 0.225). From these results we conclude that $\sigma_{w}$ and $\sigma_{z}$ are significantly greater than 0.25 ( $p$-values 0.0019 and 0.0017 on the logarithmic scale), while $\rho$ is not significantly different from zero ( $p$-value 0.5972 on the Fisher transformation scale). The absence of correlation between the random effects permits to interpret $Z_{h}$ as an NH performance measure. On the contrary, a - say - positive correlation would imply that nursing homes with a more relevant effect on transition towards critical health states are also more likely to host unhealthier patients at the first occasion. In this case, a comparison between NHs based on the $Z_{h}$ variables would not account for the different complexities nursing homes have to face at a first stage.

To understand the impact of these NH effects in terms of initial and transition probabilities, in Table 4 we report the initial probability vectors and the 180-day ahead transition matrices for the nursing homes with the higher and lower effects. Notice that these four arrays are associated to four different NHs, that is, the nursing home with the higher (lower) effect on initial probabilities is not the one with the higher (lower) effect on transition probabilities. Lower effect initial and transition probabilities are denoted by $\boldsymbol{\pi}^{-}$and $\boldsymbol{\Pi}^{-}$, while higher effect ones by $\boldsymbol{\pi}^{+}$and $\boldsymbol{\Pi}^{+}$. Each array is computed via the standard population method (Kitagawa, 1964), meaning that it is averaged across the same set of patients (and, in the case of transition matrices, across time occasions) for every NH. This is done to 


\begin{tabular}{|c|c|c|c|c|c|c|c|c|}
\hline & \multirow[b]{2}{*}{$\pi^{-}$} & & \multicolumn{6}{|c|}{$\Pi^{-}$} \\
\hline & & & 1 & 2 & 3 & 4 & 5 & 6 \\
\hline 1 & 0.189 & 1 & 0.935 & 0.064 & 0.001 & 0.000 & 0.000 & 0.000 \\
\hline 2 & 0.178 & 2 & 0.080 & 0.764 & 0.150 & 0.006 & 0.000 & 0.000 \\
\hline 3 & 0.201 & 3 & 0.002 & 0.127 & 0.681 & 0.175 & 0.014 & 0.001 \\
\hline 4 & 0.222 & 4 & 0.000 & 0.006 & 0.152 & 0.581 & 0.238 & 0.023 \\
\hline 5 & 0.211 & 5 & 0.000 & 0.001 & 0.017 & 0.194 & 0.585 & 0.203 \\
\hline \multirow[t]{3}{*}{6} & 0.000 & 6 & 0.000 & 0.000 & 0.000 & 0.000 & 0.000 & 1.000 \\
\hline & & & \multicolumn{6}{|c|}{$\Pi^{+}$} \\
\hline & $\boldsymbol{\pi}^{+}$ & & 1 & 2 & 3 & 4 & 5 & 6 \\
\hline 1 & 0.050 & 1 & 0.745 & 0.250 & 0.005 & 0.000 & 0.000 & 0.000 \\
\hline 2 & 0.068 & 2 & 0.017 & 0.505 & 0.447 & 0.028 & 0.002 & 0.000 \\
\hline 3 & 0.117 & 3 & 0.000 & 0.029 & 0.435 & 0.465 & 0.066 & 0.005 \\
\hline 4 & 0.229 & 4 & 0.000 & 0.001 & 0.035 & 0.329 & 0.529 & 0.106 \\
\hline 5 & 0.536 & 5 & 0.000 & 0.000 & 0.003 & 0.048 & 0.393 & 0.556 \\
\hline \multirow[t]{3}{*}{6} & 0.000 & 6 & 0.000 & 0.000 & 0.000 & 0.000 & 0.000 & 1.000 \\
\hline & & & \multicolumn{6}{|c|}{$\bar{\Pi}$} \\
\hline & $\bar{\pi}$ & & 1 & 2 & 3 & 4 & 5 & 6 \\
\hline 1 & 0.181 & 1 & 0.845 & 0.151 & 0.003 & 0.000 & 0.000 & 0.000 \\
\hline 2 & 0.127 & 2 & 0.082 & 0.627 & 0.271 & 0.018 & 0.001 & 0.000 \\
\hline 3 & 0.137 & 3 & 0.003 & 0.124 & 0.544 & 0.285 & 0.041 & 0.003 \\
\hline 4 & 0.180 & 4 & 0.000 & 0.007 & 0.143 & 0.447 & 0.336 & 0.066 \\
\hline 5 & 0.375 & 5 & 0.000 & 0.001 & 0.019 & 0.174 & 0.463 & 0.344 \\
\hline 6 & 0.000 & 6 & 0.000 & 0.000 & 0.000 & 0.000 & 0.000 & 1.000 \\
\hline
\end{tabular}

Table 4: Minimum effect, maximum effect and average initial and transition probabilities 
rule out the case-mix, which in this context is the effect of the different $\mathrm{NH}$ compositions with regard to patients' age and gender. The standard population is taken here to be the available sample of patients, irrespective of the NH they belong to. Finally, the overall averaged initial and transition probabilities, pooling together all NHs, denoted by $\overline{\boldsymbol{\pi}}$ and $\overline{\mathbf{\Pi}}$, are also reported in Table 4 . We recall that latent state 6 corresponds to the additional state associated to death, and many probabilities involving it are constrained to zero or one as illustrated in Section 3.2.

Looking at the overall transition probabilities, it is worth to notice that the persistence in the same state after a 180-day time period decreases for higher latent states. Beside that, the probability of worsening is greater than the probability of improving the health condition. The probability of death increases with the latent state and reaches $34.4 \%$ in latent state 5 .

As regards the NH effects on the transition probabilities, the largest effect yields the lowest persistence probabilities and the greatest probabilities of death, especially in latent state 5, compared to the least effect. In this respect we can produce a ranking of the NHs with respect to their ability in avoiding the worsening of patients' health conditions. To this purpose, we can use the scaled posterior expectation

$$
\tilde{Z}_{h}=\hat{\sigma}_{z} E\left(Z_{h} \mid \boldsymbol{X}_{h}=\boldsymbol{x}_{h}, \boldsymbol{Y}_{h}=\boldsymbol{y}_{h}\right), \quad h=1, \ldots, H
$$

Given the model assumptions, this quantity can be obtained from the approximation

$$
E\left(\boldsymbol{U}_{h} \mid \boldsymbol{X}_{h}=\boldsymbol{x}_{h}, \boldsymbol{Y}_{h}=\boldsymbol{y}_{h}\right) \approx \sum_{q=1}^{Q} \boldsymbol{\nu}_{q} \alpha_{h q}
$$

where

$$
\alpha_{h q}=\frac{P\left(\boldsymbol{Y}_{h}=\boldsymbol{y}_{h} \mid \boldsymbol{X}_{h}=\boldsymbol{x}_{h}, \boldsymbol{U}_{h}=\boldsymbol{\nu}_{q}\right) \lambda_{q}}{\sum_{q=1}^{Q} P\left(\boldsymbol{Y}_{h}=\boldsymbol{y}_{h} \mid \boldsymbol{X}_{h}=\boldsymbol{x}_{h}, \boldsymbol{U}_{h}=\boldsymbol{\nu}_{q}\right) \lambda_{q}}
$$




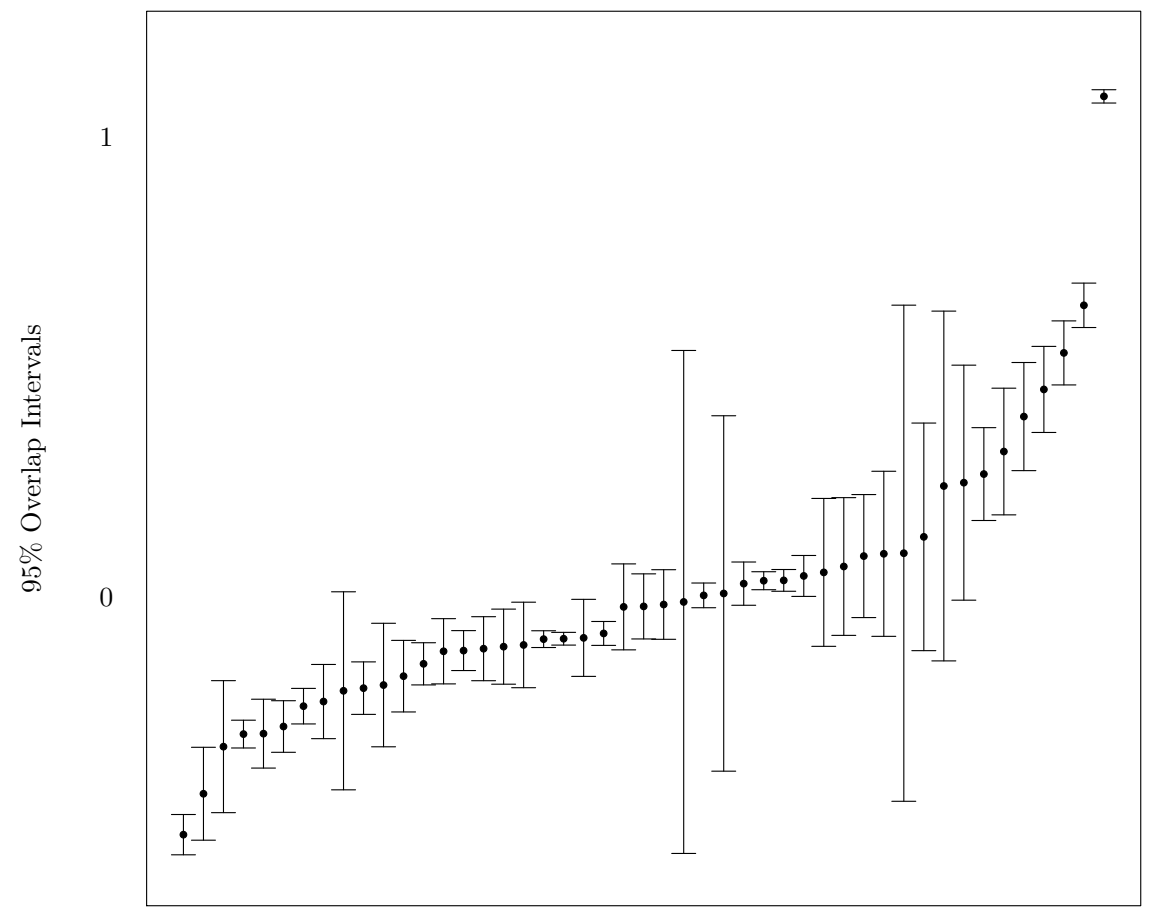

Nursing Homes

Figure 2: Posterior random effects for transition probabilities: caterpillar plot of $95 \%$ overlap intervals $(k=5)$ 
Figure 2 depicts the caterpillar plot for the values of $\tilde{Z}_{h}$. The vertical bars are the 95\% overlap intervals for pairwise comparisons: the effects on transition probabilities of two nursing homes are significantly different at the $5 \%$ level if their intervals do not overlap (Goldstein and Healy, 1995). More than 60\% of the pairwise comparisons are significantly different, denoting the importance of accounting for clustering in this application. We recall that lower values of $\tilde{Z}_{h}$ correspond to better NH performances. Notice that the worst-performing $\mathrm{NH}$, whose effect is pictured in the upper right-hand corner of Figure 2, is the one with the higher number of patients (96). Obviously, the smaller the number of units in the cluster, the larger the overlap interval.

\section{Conclusions}

In this work, we built a multilevel latent Markov model to evaluate the performance of a group of nursing homes (NHs). We constructed a NH ranking based on the capability each $\mathrm{NH}$ shows in improving or maintaining its patients in the best physical health conditions. Health status was modeled like a categorical ordinal variable. As proxies of such a variable, which is not directly observable, ten items measuring the so-called Activities of Daily Living (ADL) were used. We applied our model to a longitudinal dataset collected in the Umbria region (Italy) within the Long Term Care Facilities program, a public health protocol in which patients in nursing homes are administered a questionnaire collecting information also on the ADL. Results show that many pairwise differences between NH performances are statistically significant.

$\mathrm{NH}$ effects were modeled by means of a continuous bivariate random effect, so that the performance-based ranking can be obtained. Specifically, a normal distribution has been assumed. Although one has to bear in mind that mis-specifying the random effect 
distribution may considerably affect the results, specific reasons to discard the normality assumption do not appear to exist here. NH effects could in principle be estimated as fixed effects by introducing binary variables in the regression equations (Bartolucci et al., 2009). Within this framework, estimates are typically obtained via an Expectation Maximization (EM) algorithm. This approach has the advantage of avoiding an untestable assumption (that is, the specification of distributional form for random effects), but it is problematic in the presence of small clusters, for which the estimation process might be unstable or even unfeasible. For example, to obtain the EM estimates we used as starting point for the multilevel model (see Section 3.3), we had to aggregate nursing homes with less than ten patients.

Finally, it is worth to mention that alternative estimation methods were also proposed within this framework. These include three-step estimation (Bartolucci et al., 2015) and Bayesian estimation (Raffa and Dubin, 2015). Extending them to a multilevel context would widen the range of possible estimation methods for this kind of data. Furthermore, we argue that traditional tools to handle non-ignorable missingness - like joint models - are still not widespread in the class of latent Markov models. In our view, these represent two interesting lines of work future research in this area might be concerned with.

\section{References}

Altman, R. M. (2007). Mixed hidden Markov models: an extension of the hidden Markov model to the longitudinal data setting. Journal of the American Statistical Association 102(477), 201-210.

Bacci, S., S. Pandolfi, and F. Pennoni (2014). A comparison of some criteria for states 
selection in the latent Markov model for longitudinal data. Advances in Data Analysis and Classification 8(2), 125-145.

Bartolucci, F., A. Farcomeni, and F. Pennoni (2013). Latent Markov Models for Longitudinal Data. Statistics in the Social and Behavioural Sciences. Chapman \& Hall/CRC.

Bartolucci, F. and M. Lupparelli (2016). Pairwise likelihood inference for nested hidden Markov chain models for multilevel longitudinal data. Journal of the American Statistical Association 111(513), 216-228.

Bartolucci, F., M. Lupparelli, and G. E. Montanari (2009). Latent Markov model for longitudinal binary data: an application to the performance evaluation of nursing homes. The Annals of Applied Statistics 3(2), 611-636.

Bartolucci, F., G. E. Montanari, and S. Pandolfi (2015). Three-step estimation of latent Markov models with covariates. Computational Statistics \&3 Data Analysis 83, 287-301.

Bartolucci, F., F. Pennoni, and G. Vittadini (2011). Assessment of school performance through a multilevel latent Markov Rasch model. Journal of Educational and Behavioral Statistics 36(4), 491-522.

Baum, L. E., T. Petrie, G. Soules, and N. Weiss (1970). A maximization technique occurring in the statistical analysis of probabilistic functions of Markov chains. The Annals of Mathematical Statistics 41, 164-171.

Dempster, A. P., N. M. Laird, and D. B. Rubin (1977). Maximum likelihood from incomplete data via the EM algorithm. Journal of the Royal Statistical Society, Series B 39(1), $1-38$. 
Fisher, R. A. (1915). Frequency distribution of the values of the correlation coefficient in samples from an indefinitely large population. Biometrika 10(4), 507-521.

Fletcher, R. (1987). Practical methods of optimization (2nd ed.). New York: John Wiley \& Sons.

Gnaldi, M., S. Bacci, and F. Bartolucci (2016). A multilevel finite mixture item response model to cluster examinees and schools. Advances in Data Analysis and Classification $10(1), 53-70$.

Goldstein, H. and M. J. R. Healy (1995). The graphical presentation of a collection of means. Journal of the Royal Statistical Society, Series A 158(1), 175-177.

Goodman, L. A. (1974). Exploratory latent structure analysis using both identifiable and unidentifiable models. Biometrika 61(2), 215-231.

Gray, A. (2009). Population aging and health care expenditure. China Labor Economics 1 (10), 105-114.

Henry, K. and B. Muthén (2010). Multilevel latent class analysis: An application of adolescent smoking typologies with individual and contextual predictors. Structural Equation Modeling 17(2), 193-215.

Hirdes, J. P., G. Ljunggren, J. N. Morris, D. H. Frijters, H. Finne Soveri, L. Gray, M. Björkgren, and R. Gilgen (2008). Reliability of the interRAI suite of assessment instruments: a 12-country study of an integrated health information system. BMC Health Services Research 8(1), 277.

Kim, H., Y.-I. Jung, M. Sung, J.-Y. Lee, J.-Y. Yoon, and J.-L. Yoon (2015). Reliability 
of the interRAI Long Term Care Facilities (LTCF) and interRAI Home Care (HC). Geriatrics $\&$ Gerontology International 15(2), 220-228.

Kitagawa, E. M. (1964). Standardized comparisons in population research. Demography 1(1), 296-315.

Koukounari, A., I. Moustaki, N. C. Grassly, I. M. Blake, M.-G. Basáñez, M. Gambhir, D. C. Mabey, R. L. Bailey, M. J. Burton, and A. W. Solomon (2013). Using a nonparametric multilevel latent Markov model to evaluate diagnostics for trachoma. American Journal of Epidemiology 177(9), 913-922.

Little, R. J. and D. B. Rubin (2002). Statistical Analysis with Missing Data (2 ${ }^{\text {nd }}$ ed.). Wiley.

Makai, P., W. B. Brouwer, M. A. Koopmanschap, E. A. Stolk, and A. P. Nieboer (2014). Quality of life instruments for economic evaluations in health and social care for older people: a systematic review. Social Science 85 Medicine 102, 83-93.

Maruotti, A. (2011). Mixed hidden Markov models for longitudinal data: an overview. International Statistical Review 79(3), 427-454.

Maruotti, A. and R. Rocci (2012). A mixed nonhomogeneous hidden Markov model for categorical data, with application to alcohol consumption. Statistics in Medicine 31(9), $871-886$.

Montanari, G. E. and S. Pandolfi (2016). Evaluation of health care services through a latent Markov model with covariates. In SIS 2016 - $48^{\text {th }}$ Scientific Meeting of the Italian Statistical Society. 
Montanari, G. E., M. G. Ranalli, and P. Eusebi (2010). Multilevel latent class models for evaluation of long-term care facilities. In Data Analysis and Classification, pp. 249-256. Springer.

Oehlert, G. W. (1992). A note on the delta method. The American Statistician 46(1), $27-29$.

Press, W. H., B. P. Flannery, S. A. Teukolsky, and W. T. Vetterling (1989). Numerical recipes, Volume 3. Cambridge University Press, Cambridge.

Raffa, J. D. and J. A. Dubin (2015). Multivariate longitudinal data analysis with mixed effects hidden Markov models. Biometrics $71(3), 821-831$.

Schwarz, G. (1978). Estimating the dimension of a model. The Annals of Statistics 6(2), 461-464.

Stephens, M. (2000). Dealing with label switching in mixture models. Journal of the Royal Statistical Society, Series B 62(4), 795-809.

Vermunt, J. (2003). Multilevel latent class models. Sociological Methodology 33(1), 213239.

Vermunt, J. K., R. Langeheine, and U. Bockenholt (1999). Discrete-time discrete-state latent Markov models with time-constant and time-varying covariates. Journal of Educational and Behavioral Statistics 24(2), 179-207.

Welch, L. R. (2003). Hidden Markov models and the Baum-Welch algorithm. IEEE Information Theory Society Newsletter 53, 1-13. 
White, C. (2007). Health care spending growth: how different is the United States from the rest of the OECD? Health Affairs 26(1), 154-161.

Wiggins, L. M. (1973). Panel analysis: Latent probability models for attitude and behavior processes. Jossey-Bass. 


\section{A multilevel latent Markov, model for the evaluation of nursing homes' performance: Supplementary material}




\section{Log-likelihood gradient}

The approximate gradient of the log-likelihood function is given by

$$
\frac{\partial \ell(\boldsymbol{\theta})}{\partial \boldsymbol{\theta}} \approx \sum_{h=1}^{H} \frac{1}{P\left(\boldsymbol{Y}_{h}=\boldsymbol{y}_{h} \mid \boldsymbol{X}_{h}=\boldsymbol{x}_{h}\right)} \sum_{q=1}^{Q}\left[\exp \left(\sum_{i=1}^{n_{h}} \ell_{h i q}(\boldsymbol{\theta})\right)\left(\sum_{i=1}^{n_{h}} \frac{\partial \ell_{h i q}(\boldsymbol{\theta})}{\partial \boldsymbol{\theta}}\right) \lambda_{q}\right],
$$

where $\ell_{h i q}(\boldsymbol{\theta})=\log P\left(\boldsymbol{Y}_{h i}=\boldsymbol{y}_{h i} \mid \boldsymbol{X}_{h i}=\boldsymbol{x}_{h i}, \boldsymbol{U}_{h}=\boldsymbol{\nu}_{q}\right)$. Given the complete log-likelihood

$$
\ell_{h i q}^{*}(\boldsymbol{\theta})=\log P\left(\boldsymbol{Y}_{h i}=\boldsymbol{y}_{h i}, \boldsymbol{V}_{h i}=\boldsymbol{v}_{h i} \mid \boldsymbol{X}_{h i}=\boldsymbol{x}_{h i}, \boldsymbol{U}_{h}=\boldsymbol{\nu}_{q}\right)
$$

and its posterior expectation $E_{h i q}^{*}(\boldsymbol{\theta})=E\left(\ell_{h i q}^{*}(\boldsymbol{\theta}) \mid \boldsymbol{Y}_{h i}=\boldsymbol{y}_{h i}\right)$, a theoretical result (Oakes, 1999) stating that

$$
\frac{\partial \ell_{h i q}(\boldsymbol{\theta})}{\partial \boldsymbol{\theta}}=\frac{\partial E_{h i q}^{*}(\boldsymbol{\theta})}{\partial \boldsymbol{\theta}}
$$

is exploited to compute each derivative present in (1).

\section{Results for the model with $k=6$}

In this section, we report the results for the model with $k=6$. As stated in the paper, these are not substantially different from those obtained in the model with $k=5$, denoting the stability of the conclusions drawn. Table 1 reports estimates and standard errors for the same parameters we consider in the model with five latent states. A comparison with paper's Table 3 shows that both estimates and standard errors are very similar in the two models. Parameters' significance and interpretation remain unchanged.

Figure 1 reports the normalized item scores. Again, the overall trend is very close to that of the model with $k=5$ (paper's Figure 1). Specifically, once more we observe that for every latent state $v$ the biggest difficulties concern items 1 and 4 (washing/taking a shower and dressing the lower part of the body), while the easier activities are bed mobility 


\begin{tabular}{ccccccccc}
\hline & $\beta_{1}$ & $\beta_{2}$ & $\gamma_{1}$ & $\gamma_{2}$ & $\gamma_{3}$ & $\sigma_{w}$ & $\sigma_{z}$ & $\rho$ \\
\hline est. & 0.032 & 0.482 & 0.020 & -0.262 & -0.004 & 0.469 & 0.372 & -0.131 \\
s.e. & 0.006 & 0.121 & 0.004 & 0.095 & 0.001 & 0.102 & 0.056 & 0.220 \\
\hline
\end{tabular}

Table 1: Main parameter estimates and standard errors for the model with $k=6$

and eating (items 9 and 10). Figure 2 depicts the caterpillar plot of the scaled posterior

expectations $\tilde{Z}_{h}$. The resulting ranking is almost identical to the previous one, with a Spearman correlation coefficient of 0.995 . Finally, Table 2 is the analogous for $k=6$ of paper's Table 4 . Once again, results are very similar overall.

\section{References}

Oakes, D. (1999). Direct calculation of the information matrix via the em. J. R. Stat. Soc. Ser. B Stat. Methodol. 61(2), 479-482. 


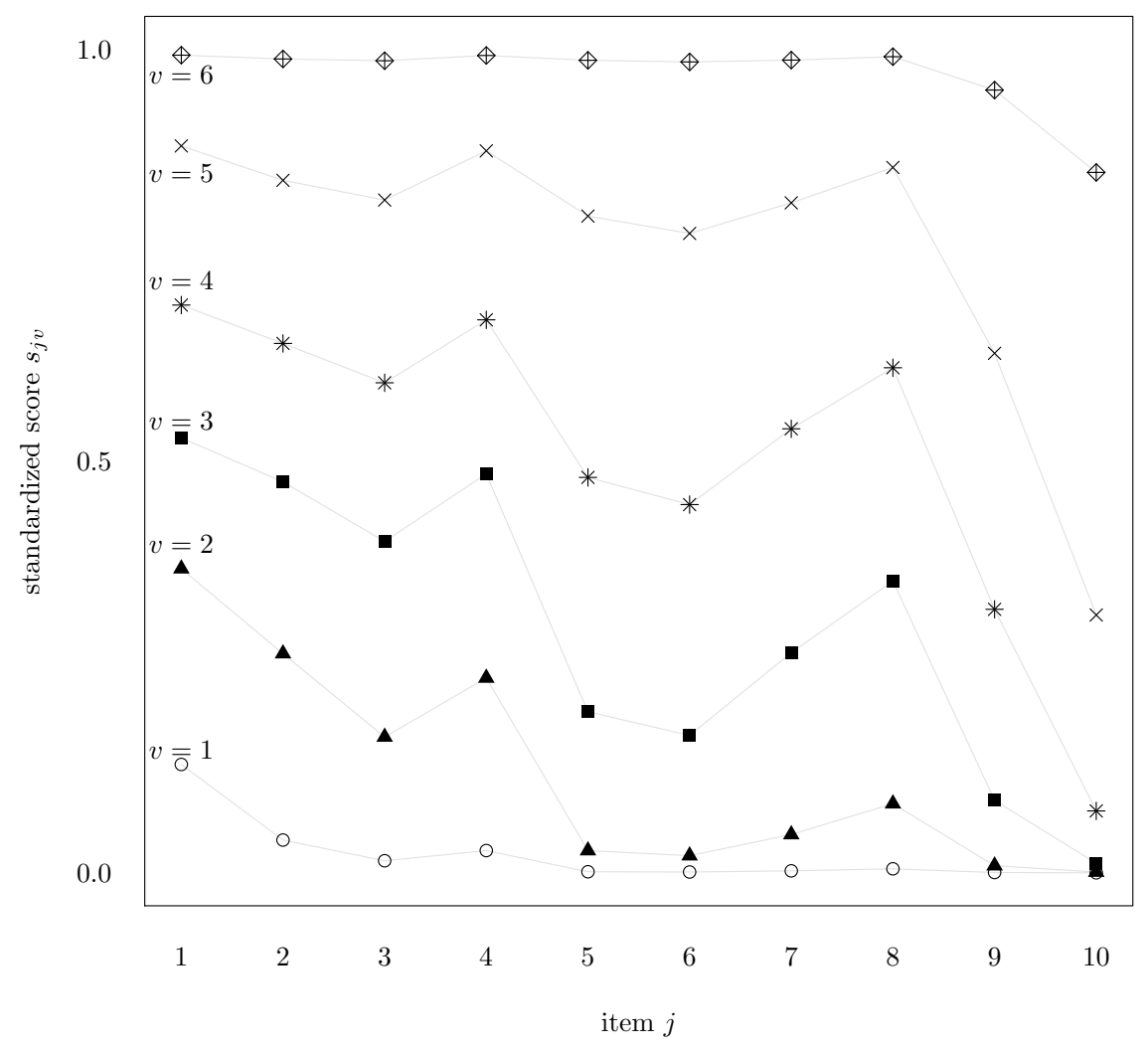

Figure 1: Normalized item scores $s_{j v}(k=6)$ 


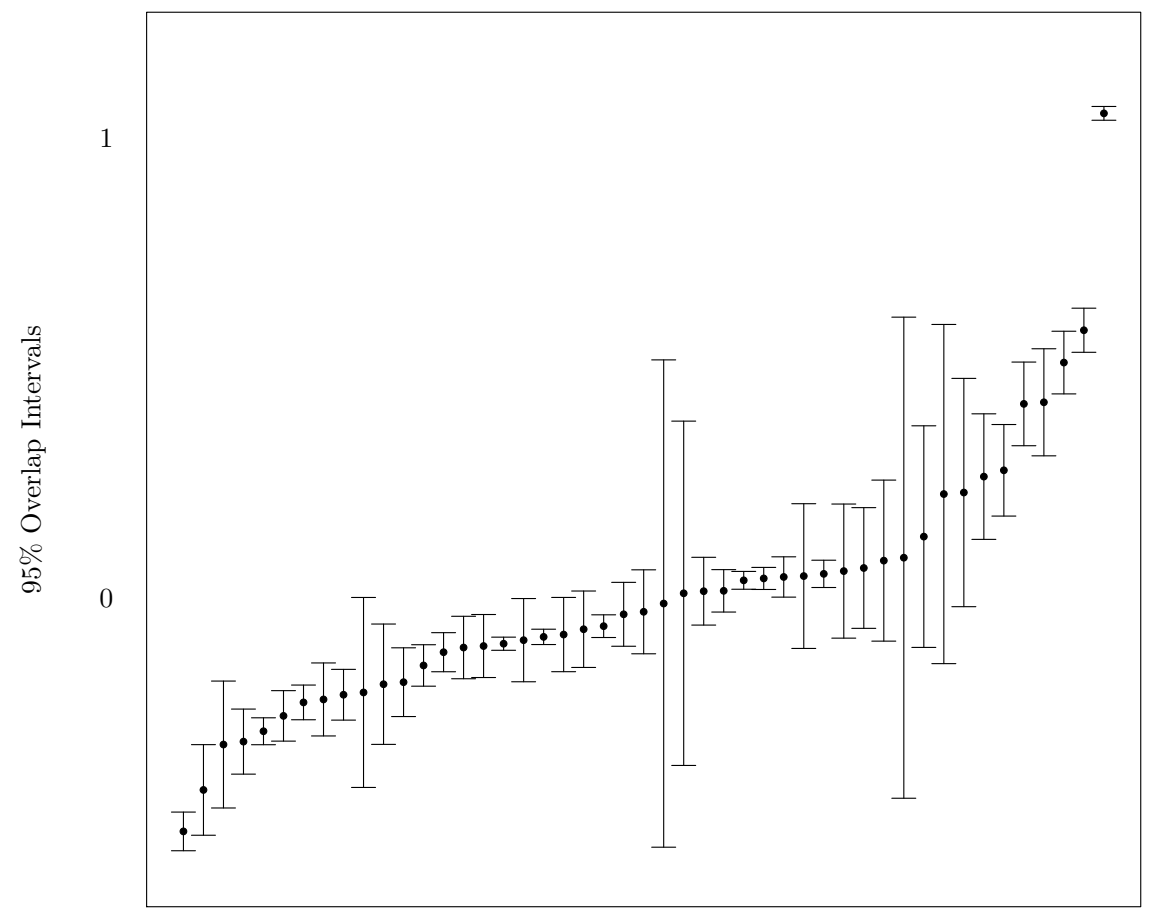

Nursing Homes

Figure 2: Posterior random effects for transition probabilities: caterpillar plot of $95 \%$ overlap intervals $(k=6)$ 


\begin{tabular}{|c|c|c|c|c|c|c|c|c|c|}
\hline & \multirow[b]{2}{*}{$\pi^{-}$} & & \multicolumn{7}{|c|}{$\Pi^{-}$} \\
\hline & & & 1 & 2 & 3 & 4 & 5 & 6 & 7 \\
\hline 1 & 0.103 & 1 & 0.924 & 0.076 & 0.000 & 0.000 & 0.000 & 0.000 & 0.000 \\
\hline 2 & 0.139 & 2 & 0.046 & 0.854 & 0.096 & 0.004 & 0.000 & 0.000 & 0.000 \\
\hline 3 & 0.160 & 3 & 0.001 & 0.112 & 0.673 & 0.200 & 0.013 & 0.001 & 0.000 \\
\hline 4 & 0.177 & 4 & 0.000 & 0.007 & 0.157 & 0.624 & 0.194 & 0.018 & 0.001 \\
\hline 5 & 0.214 & 5 & 0.000 & 0.000 & 0.010 & 0.154 & 0.566 & 0.244 & 0.025 \\
\hline 6 & 0.206 & 6 & 0.000 & 0.000 & 0.001 & 0.018 & 0.196 & 0.580 & 0.206 \\
\hline \multirow[t]{3}{*}{7} & 0.000 & 7 & 0.000 & 0.000 & 0.000 & 0.000 & 0.000 & 0.000 & 1.000 \\
\hline & & & \multicolumn{7}{|c|}{$\Pi^{+}$} \\
\hline & $\boldsymbol{\pi}^{+}$ & & 1 & 2 & 3 & 4 & 5 & 6 & 7 \\
\hline 1 & 0.024 & 1 & 0.719 & 0.279 & 0.002 & 0.000 & 0.000 & 0.000 & 0.000 \\
\hline 2 & 0.042 & 2 & 0.010 & 0.646 & 0.326 & 0.017 & 0.001 & 0.000 & 0.000 \\
\hline 3 & 0.066 & 3 & 0.000 & 0.026 & 0.412 & 0.499 & 0.058 & 0.004 & 0.000 \\
\hline 4 & 0.107 & 4 & 0.000 & 0.001 & 0.038 & 0.401 & 0.476 & 0.078 & 0.006 \\
\hline 5 & 0.225 & 5 & 0.000 & 0.000 & 0.002 & 0.038 & 0.326 & 0.525 & 0.109 \\
\hline 6 & 0.536 & 6 & 0.000 & 0.000 & 0.000 & 0.004 & 0.051 & 0.396 & 0.549 \\
\hline \multirow[t]{3}{*}{7} & 0.000 & 7 & 0.000 & 0.000 & 0.000 & 0.000 & 0.000 & 0.000 & 1.000 \\
\hline & & & \multicolumn{7}{|c|}{$\bar{\Pi}$} \\
\hline & $\bar{\pi}$ & & 1 & 2 & 3 & 4 & 5 & 6 & 7 \\
\hline 1 & 0.111 & 1 & 0.829 & 0.170 & 0.001 & 0.000 & 0.000 & 0.000 & 0.000 \\
\hline 2 & 0.111 & 2 & 0.049 & 0.742 & 0.198 & 0.011 & 0.001 & 0.000 & 0.000 \\
\hline 3 & 0.110 & 3 & 0.001 & 0.110 & 0.537 & 0.312 & 0.037 & 0.003 & 0.000 \\
\hline 4 & 0.120 & 4 & 0.000 & 0.007 & 0.146 & 0.496 & 0.297 & 0.049 & 0.004 \\
\hline 5 & 0.174 & 5 & 0.000 & 0.000 & 0.011 & 0.143 & 0.439 & 0.339 & 0.068 \\
\hline 6 & 0.373 & 6 & 0.000 & 0.000 & 0.001 & 0.020 & 0.173 & 0.463 & 0.343 \\
\hline 7 & 0.000 & 7 & 0.000 & 0.000 & 0.000 & 0.000 & 0.000 & 0.000 & 1.000 \\
\hline
\end{tabular}

Table 2: Minimum effect, maximum effect and average initial and transition probabilities $(k=6)$ 